\title{
Characterization of mind wandering using fNIRS
}

\author{
Gautier Durantin ${ }^{1,2,3 *}$, Frederic Dehais ${ }^{1}$ and Arnaud Delorme ${ }^{2,3}$ \\ ${ }^{1}$ Département Conception des Véhicules Aérospatiaux, Institut Supérieur de l'Aéronautique et de l'Espace, Toulouse, France, \\ ${ }^{2}$ Centre de Recherche Cerveau et Cognition, Universite de Toulouse, UPS, Toulouse, France, ${ }^{3} \mathrm{CNRS}$, CerCo, \\ Toulouse, France
}

OPEN ACCESS

Edited by:

Mikhail Lebedev, Duke University, USA

Reviewed by: Konstantinos Priftis, University of Padova, Italy Mehdi Ordikhani-Seyedlar, Technical University of Denmark,

Denmark Ling-Chia Chen,

University of Oldenburg, Germany

*Correspondence: Gautier Durantin, Département Conception des Véhicules Aérospatiaux, Institut Supérieur de

l'Aéronautique et de l'Espace, 10 Avenue Edouard Belin, 31055 Toulouse, France gautier.durantin@isae.fr

Received: 26 January 2015 Accepted: 06 March 2015 Published: 26 March 2015

Citation:

Durantin G, Dehais F and Delorme A

(2015) Characterization of mind wandering using fNIRS.

Front. Syst. Neurosci. 9:45. doi: 10.3389/fnsys.2015.00045
Assessing whether someone is attending to a task has become important for educational and professional applications. Such attentional drifts are usually termed mind wandering (MW). The purpose of the current study is to test to what extent a recent neural imaging modality can be used to detect MW episodes. Functional near infrared spectroscopy is a non-invasive neuroimaging technique that has never been used so far to measure MW. We used the Sustained Attention to Response Task (SART) to assess when subjects attention leaves a primary task. Sixteen-channel fNIRS data were collected over frontal cortices. We observed significant activations over the medial prefrontal cortex (mPFC) during MW, a brain region associated with the default mode network (DMN). fNIRS data were used to classify MW data above chance level. In line with previous brain-imaging studies, our results confirm the ability of fNIRS to detect Default Network activations in the context of MW.

Keywords: mind wandering, fNIRS, linear discriminant analysis, default mode network

\section{Introduction}

While reading books, people's attention may drift towards self-centered matters. After some time, the readers may realize that they have lost track of their reading and have started to mind wander. These attentional drifts are called mind wandering (MW) episodes and people are generally unaware of when they occur. Avoiding these attentional drifts is not only a matter of will power since even after years of practice focused meditation, meditators still experience these drifts regardless of their efforts to avoid them (Braboszcz et al., 2010). The assessment of MW events may be of importance for several potential applications. Since attentional deficit/hyperactivity disorder (ADHD) patients also have higher occurrence of MW episodes (Peterson et al., 2009), determining the amount of MW would be beneficial for those individuals. This could also have benefits for the science of education since MW may prevent the assimilation of educational material (Szpunar et al., 2013). Finally, as MW reduces the cortical processing of the external environment (Mooneyham and Schooler, 2013), it may jeopardize safety in operational situations such as driving a vehicle (He et al., 2011; Galéra et al., 2012) or flying a plane (Casner and Schooler, 2014).

Studies on MW have usually involved sustained attention paradigms such as breath counting (Braboszcz et al., 2010) or go-no go tasks (Shaw et al., 2013). One example of a go-no go task includes the popular Sustained Attention to Response Task (SART), wherein single digit numbers appear one at a time on a computer screen: subjects are instructed to press a button whenever a number other than the target number (3) appears (Manly et al., 1999). The SART is simple so subjects' attention frequently leaves the primary task, in addition to being sensitive to the tendency for participants to automate their behavior. During episodes of MW, subjects tend to press the button systematically (even when three 
appears). By counting the number of successive missed targets, it is possible to assess the time and frequency of MW episodes. As outlined in Smallwood and Schooler (Smallwood and Schooler, 2006), investigations of the experience of MW during the SART support that, first, blocks in which this phenomenon occurs are associated with faster response times than are blocks in which attention is directed towards the task. Second, high levels of MW reported with retrospective questionnaires are associated with a tendency to make an error during periods of task disengagement. We will primarily focus on the second point-errors during the SART-to process INIRS data.

Due to its importance, studies aiming at characterizing MW using various objective psychophysiological measurements have flourished in the last years (Smallwood and AndrewsHanna, 2013). For instance, findings from a study (Smallwood et al., 2008) in which participants received experience sampling probes while performing the SART showed that the amplitude of a late positive ERP component of the event related potentials measured by EEG (known as P3) was reduced by MW, confirming the hypothesis that MW induces decoupling from the environment. Christoff et al. (2009) investigated functional Magnetic Resonance Imaging (fMRI) samples while participants were engaged in the SART task. Interestingly, they found evidence of activation of Default Mode Network (DMN)-known to exert high activity levels during off-task conditions-and recruitment of executive network regions during MW episodes. Numerous other studies using sustained attention tasks, showed a greater implication of the right hemisphere in the process of sustained attention (Warm et al., 2009; Stevenson et al., 2011).

However, the importance of MW phenomenon both in laboratory experiments and in daily life (Killingsworth and Gilbert, 2010; Mooneyham and Schooler, 2013) calls for means to characterize further its neurophysiological correlates in reallife conditions using portable solutions.

Functional near infra-red spectroscopy (fNIRS) is a noninvasive neuroimaging technique that is portable, relatively low-cost and has high spatial resolution, which makes it a promising technique for research (Strait and Scheutz, 2014). fNIRS has successfully been used for the monitoring of attentional states (Harrivel et al., 2013), vigilance (Warm et al., 2009; Helton et al., 2013) and task unrelated thoughts (Stevenson et al). In this study, we used the fNIRS in a SART task as a mean to characterize MW with a classification approach. The objective of this experiment is to assess fNIRS sensitivity to measure neural correlates of $\mathrm{MW}$, and to discriminate single trial MW vs. non-MW episodes using formal classification.

\section{Material and Methods}

\section{Participants}

The experiment was approved by local ethics committee (Comité de Protection des Personnes). Twenty-three male students from ISAE school of engineering gave their consent to participate in the experiment (21 right-handed; age range 21-24 years, mean age: 22.6). All participants reported normal or corrected vision, and none were suffering from neuropsychological problems.

Prior to the experiment, subjects were provided instructions for the SART task. No explanations were given about the phenomenon of MW, in order to avoid this having an impact on their performance.

\section{SART}

Subjects were asked to perform a computerized SART task. The SART task consists of a simple go/no-go task in which a single infrequent target digit is presented (here the digit 3) amongst frequent non-targets digits (1-9). The computer screen was placed approximately $70 \mathrm{~cm}$ from the participants' head. Each digit was presented for $500 \mathrm{~ms}$ on the computer screen and then replaced by a fixation mark (" $X$ ") for $1000 \mathrm{~ms}$ (see Figure 1). Digits appeared in white on a black background and were approximately $3 \mathrm{~cm}$ high in Arial font. The participants were asked to press the spacebar of the computer keyboard for non-target digits, and not to press it if the digit was a target (3). The target trials for which the participants inaccurately pressed the spacebar were considered "SART Errors". In the rest of this manuscript, we will designate by the term "SART No errors" the other target trials.

The experiment consisted of the presentation of two blocks of 198 trials, 22 of them (11\%) being target trials. Targets presentation was pseudorandom, and ensured that two target trials would not follow each other. The mean interval between two target trials was $11.6 \mathrm{~s}$ (SE: $3.35 \mathrm{~s}$ ). Preliminary to the experiment, for training purposes, subjects performed 18 practice trials ( 2 of which were targets). Subjects were told to respond as accurately and as quickly as possible to digit presentations.

At the end of the experiment, subjects were asked to complete a questionnaire where they could report their opinion about the task and whether they experienced any difficulties. In the

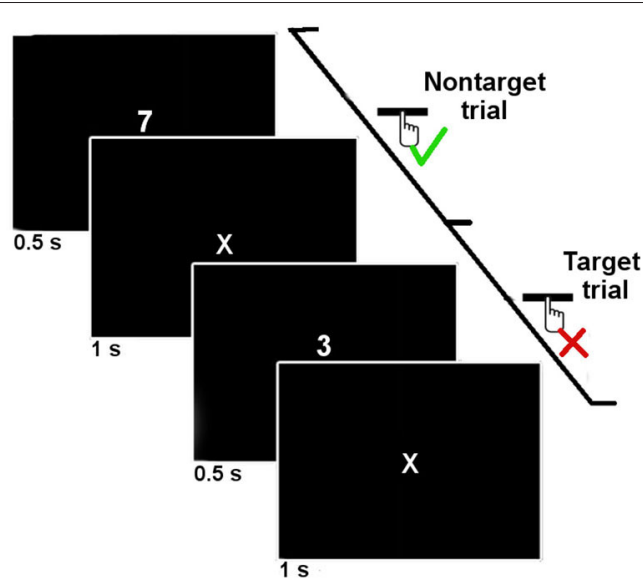

FIGURE 1 | Time course of two trials of the SART protocol. The digits were presented $500 \mathrm{~ms}$ with an onset-to-onset interval of $1500 \mathrm{~ms}$. Subjects were asked to press the spacebar when a non-target digit was presented, and not to respond when a target digit (3) was presented. 
questionnaire, we also asked subjects whether they had any thoughts that were unrelated to the task (Gruberger et al., 2011).

\section{Data Acquisition}

During the experiment, hemodynamic data from the prefrontal cortex were recorded using a fNIR100 device (Biopac Inc.). The device consists of four light-emitting diodes (LED) sources of $730 \mathrm{~nm}$ and $850 \mathrm{~nm}$ (LED current: $12 \mathrm{~mA}$ ), and ten detectors (see Figure 2 for arrangement). The source and detectors are separated by $2.5 \mathrm{~cm}$, resulting in 16 optodes uniformly placed on a rectangular grid on the forehead (see Figure 2). Data was collected with a sampling frequency of $2 \mathrm{~Hz}$. A baseline of $10 \mathrm{~s}$ at the beginning of the experiment was used to calibrate the device. Optodes 1, 3 and 5 were defective and removed from all subjects.

\section{Data Processing}

The 95\% confidence intervals for the number of SART errors committed during the task were calculated using the SingleBayes method from Crawford and Garthwaite (Crawford and Garthwaite, 2007), and the probability density function of the apparition of SART errors during the experiment was estimated using Statistica ${ }^{\oplus}$, using periods of $30 \mathrm{~s}$ and Weibull model estimation, a probability distribution commonly used in survival analysis for event density probability function estimation (Kennedy and Gehan, 1971).
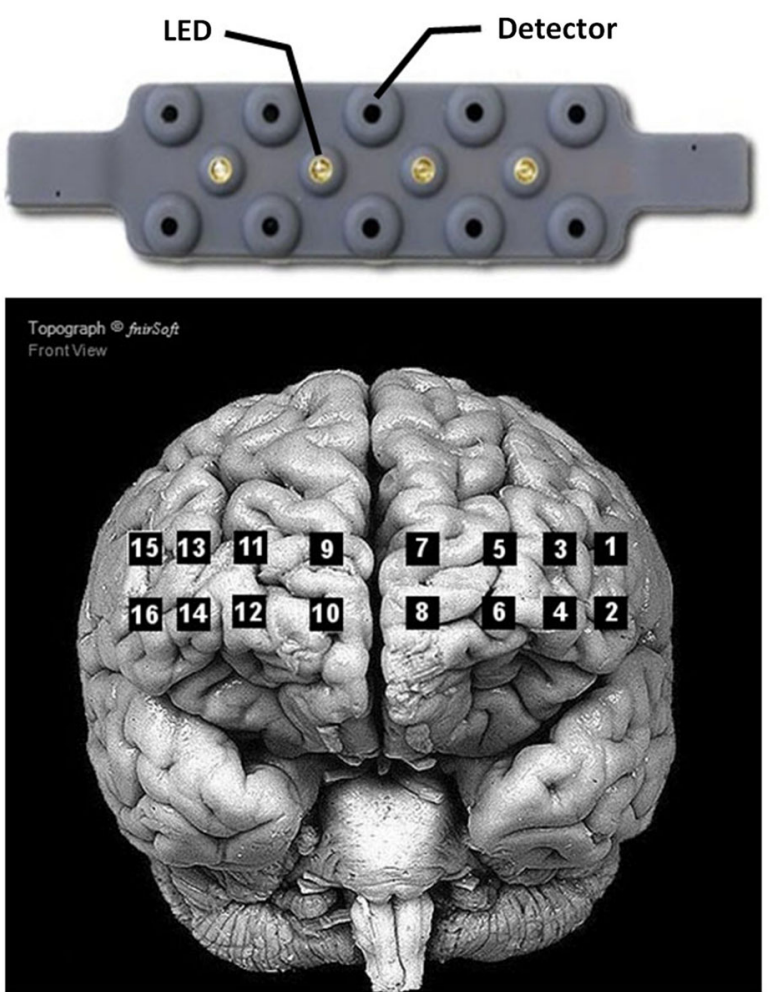

FIGURE 2 | Arrangement of the 4 LED sources and 10 detectors of the Biopac ${ }^{\circledR}$ fNIR100 device (top), and location of the corresponding optodes on the cortex (bottom). Adapted from fnirSoft ${ }^{\circledR}$ software for NIRS data analyses.
The density function is defined as follow:

$$
p d f(t)=\frac{P(t<S A R T \text { Error }<t+d t)}{d t}
$$

Hemodynamic data recorded by fNIRS were processed using the EEGLAB toolbox for MatLab ${ }^{\oplus}$ (Delorme and Makeig, 2004). Continuous data were high-pass filtered using a short non-linear Infinite Impulse Response (IIR) filter of order 6 and a cutoff frequency of $0.02 \mathrm{~Hz}$. We extracted epochs from continuous data for the SART Error and SART No Error conditions, starting $30 \mathrm{~s}$ before stimulus apparition, and stopping $10 \mathrm{~s}$ after. The significance of the hemodynamic changes mapped using fNIRS was tested at the topographical level, using the Montecarlo Statistics and using the Cluster Correction for multiple comparisons (Maris and Oostenveld, 2007) to correct for multiple optodes measurements.

\section{Classification}

We used Linear Discriminant Analysis (LDA) for the classification of MW epochs as compared to epochs in which subjects were concentrated. Non-target epochs were not considered here. We considered only MW epochs, with either a response (correct detection) or no response (incorrect detection). LDA is a basic classifier that is robust and fast to compute. We used a 10-fold cross-validation approach to test our model. This means that there are 10 iterations to the algorithm. For each iteration, $90 \%$ of the data is used for learning the LDA weights and the remaining $10 \%$ of the data is used for testing the model the data for testing is always different from the previous iterations.

\section{Results}

\section{Behavioral Results}

Over the 44 target trials of the SART task, the participants made a mean of 12.7 errors (standard deviation: 7.0), which represents $29 \%$ of the target trials. Figure 3 shows the probability density function of the apparition of SART errors during the two sessions of the experiment. This figure exhibits the increasing SART Errors density over time.

At the end of the experiment, 19 out of the 23 participants stated in the questionnaires that they found it difficult to stay focused on the task and had irrelevant thoughts during the experiment. The four subjects who did not mention this fact committed an average of 10.8 errors (SD: 6.45), whereas the 19 subjects conscious of MW committed on average 13.1 errors (SD: 7.37). Although the small sample size for the first group of four subjects did not permit group level statistical testing, the individual comparison of each of these subjects with the group of subjects who reported MW revealed that none of the subjects who were not aware of MW committed significantly less SART Errors than the 19 others.

\section{Hemodynamics}

Figure 4 shows the topography of $\mathrm{HbO}_{2}$ concentration under both SART Error and SART No Error conditions, from $15 \mathrm{~s}$ until 


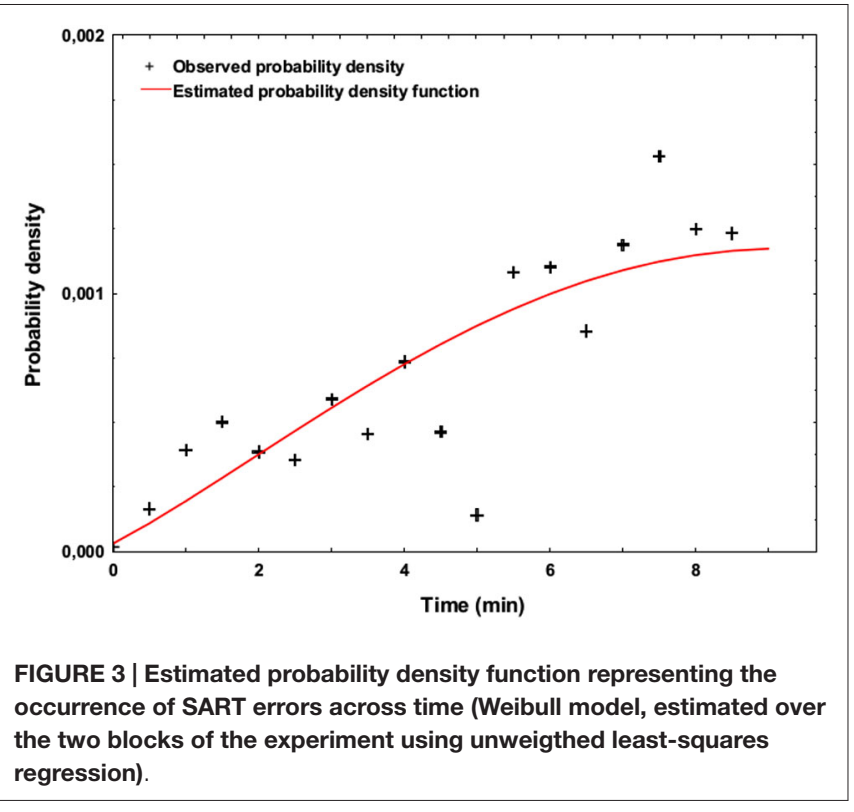

$5 \mathrm{~s}$ before the apparition of the stimulus. After correcting for multiple comparisons, our analysis revealed significantly higher levels of oxygenated hemoglobin for optodes 7, 9 and 11, located in the dorsomedial prefrontal cortex, preceding SART Errors (associated with MW episodes). Figure 5 indicates the temporal dynamics associated with optode 9 , and shows that the greater activation observed in the medial prefrontal cortex (mPFC) before SART Errors returns to normal before the arrival of the stimulus. No significant variations relative to SART Errors were found on the deoxy-hemoglobin $(\mathrm{HHb})$ signal.

\section{Classification}

We used data from only 11 subjects to classify SART Error and SART No Error trials, keeping only the subjects who made at least 10 errors, so we would have enough trials to train and test the classifier. Out of the 11 subjects, 7 of them had a classification accuracy superior to $60 \%$. A Wilcoxon sign test showed that this result was unlikely to occur by chance $(p<0.016$; degree of freedom of 10). Results are summarized in Table 1, and Figure 6 shows the individual accuracy results.

\section{Discussion}

We showed that MW occurred during the SART, as 19 out of 23 participants retrospectively reported MW meta-awareness during the experiment (Smallwood and Schooler, 2006). The high number of errors made during target trials is consistent with SART characteristics regarding MW (Manly et al., 1999), and notably the increasing occurrence of MW episodes with the time spent on the task as shown in Figure 3, are consistent with previous findings using sustained attention tasks (Mackworth, 1948; Allan Cheyne et al., 2009). However, contrary to Smallwood and Schooler's study, the retrospective awareness of MW could not be associated with a higher number of SART errors. Although 4 out of 23 subjects did not report experiencing $\mathrm{MW}$, it is noticeable this was not associated with the absence of SART Errors (mean number of errors: 10.8), therefore showing that SART Errors occurred during the experiment, even without MW meta-awareness.

Our results showed that it is possible to discriminate responses where subjects are not paying attention to the task, compared to periods where they are attending based solely on the fNIRS signal. We observed significant differences in the fNIRS signal measured in the mPFC preceding the cue presentation for correct vs. incorrect trials. Notably, the medial prefrontal activations we observed prior to SART errors (see Figure 4) were similar to activations observed previously in fMRI with the same task (Christoff et al., 2009). These results, in conjunction with previous neuroimaging MW investigations (Mason et al., 2007), support the feasibility of using fNIRS to detect DMN activity (Sasai et al., 2012), and its implication in the phenomenon of MW. Our results did not show implication of the Executive Network areas such as dorsolateral prefrontal cortex (DLPFC) during SART errors, as revealed by two previous fMRI studies (Christoff et al., 2009; Stawarczyk et al., 2011). In these studies, DLPFC activation was related to MW meta-awareness but not to SART Errors. However, our experimental paradigm was not designed to measure MW meta-awareness and thus did not allow to state about DLPFC activations. Moreover, the absence of left

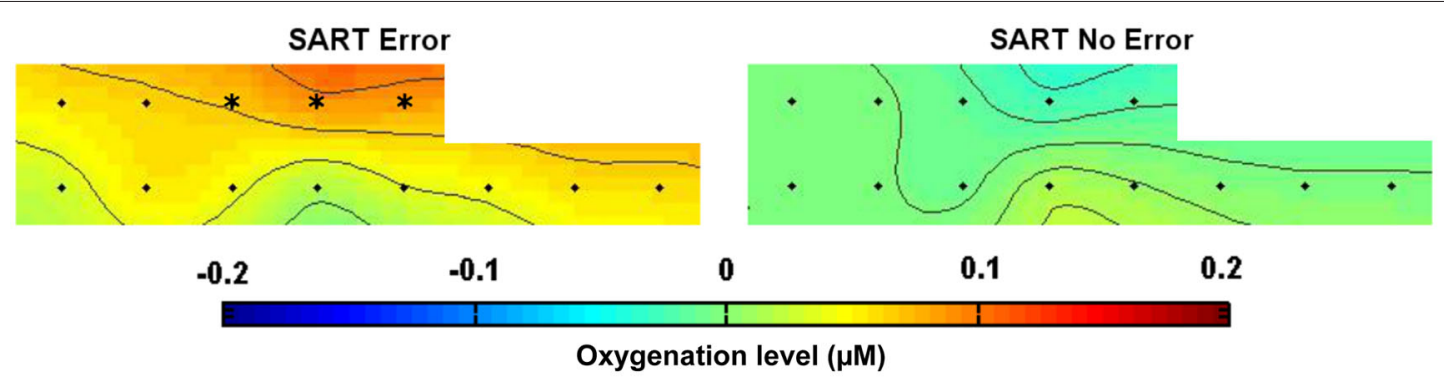

FIGURE 4 | Topography of $\mathrm{HbO}_{2}$ concentration over the prefrontal cortex during SART Error (on the left) and SART No Error (on the right) conditions, averaged [-15 s; $\mathbf{- 5} \mathbf{s}$ ] before the apparition of the target stimulus across all subjects. The color code represents the level of $\mathrm{HbO} \mathrm{O}_{2} \mathrm{concentration}$ changes relative to baseline (in $\mu \mathrm{M}$ ). Optodes exhibiting significant differences (all in the $\mathrm{mPFC}$ ) are marked with a * (significance level $=0.01$ after correction for multiple comparisons). 


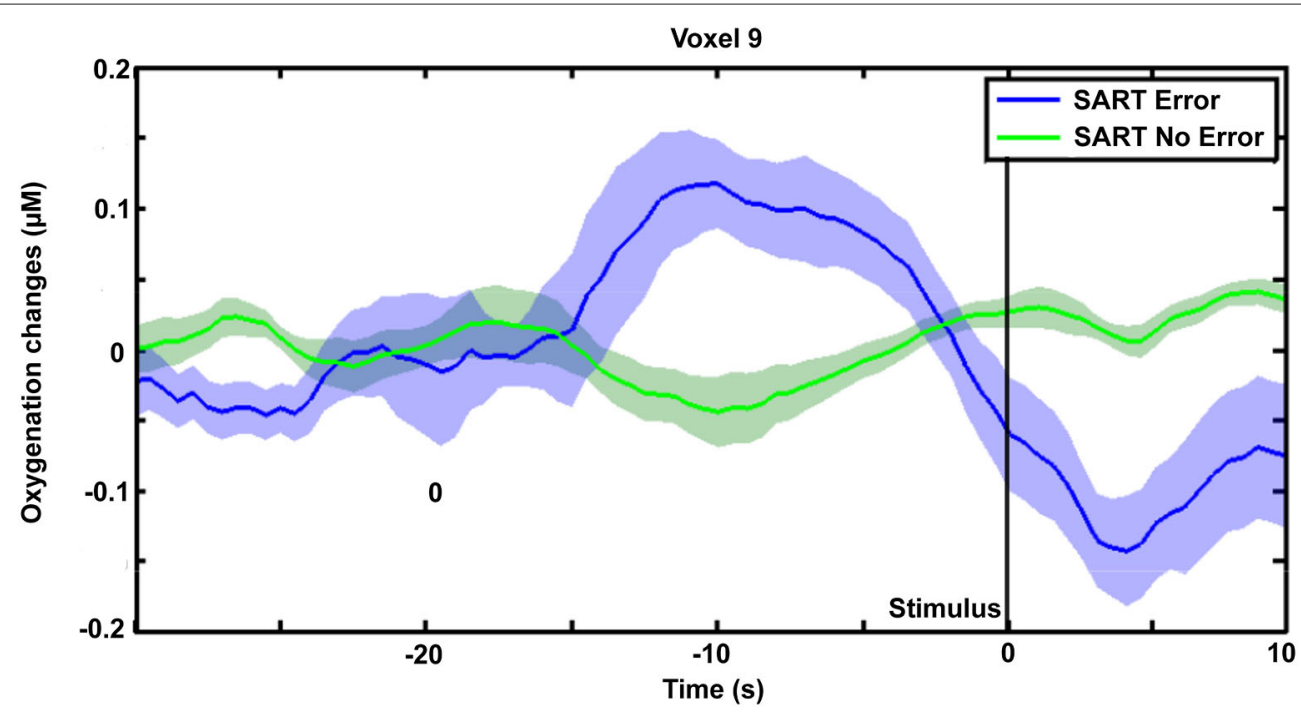

FIGURE 5 | Variation of $\mathrm{HbO}_{2}$ concentration (averaged trials across subjects) on optode 9 (in the mPFC) preceding SART Error (in blue) and SART No Error trials (in green). Shaded areas represent the standard error of the mean for each condition.

DLPFC activations could be due to the fact that the optodes in this area were defective and had to be removed from the study.

An important contribution of our study concerns the temporal dynamics of the activations observed in the $\mathrm{MPFC}$. This area did not show sustained activation during MW episodes, as the level of $\mathrm{HbO}_{2}$ measured faded even before target stimulus apparition (see. Figure 5). Similarly, previous investigation of MW using SART protocol in fMRI only found DMN activations preceding MW occurrences (Christoff et al., 2009; Stawarczyk et al., 2011). This would suggest that the contribution of the $\mathrm{mPFC}$ to the DMN is the strongest at the beginning of the MW episode, which would be consistent with the role of MPFC during sleep as shown by functional connectivity MRI studies (Sämann et al., 2011). Hence, this transient activation of the mPFC could suggest that this brain area plays a role to switch from a concentrated to a MW state. However, further investigation using self-caught MW protocols and analyzing the temporal dynamics of brain activation would be needed to support this hypothesis.

Some limitations remain concerning the interpretation of SART Errors. Although previous research has demonstrated that performance on the SART task is mainly determined by the capacity to endogenously sustain attention (Manly et al., 1999), there remains an ongoing debate as to whether SART errors could be related to impulsivity in subjects' responses (Helton

TABLE 1 | Mean mind wandering episodes classification performance across subjects.

\begin{tabular}{lcc}
\hline & Mean & Standard error \\
\hline Accuracy & $56 \%$ & $2.70 \%$ \\
Sensitivity & $52 \%$ & $6.33 \%$ \\
Specificity & $62 \%$ & $4.82 \%$ \\
\hline
\end{tabular}

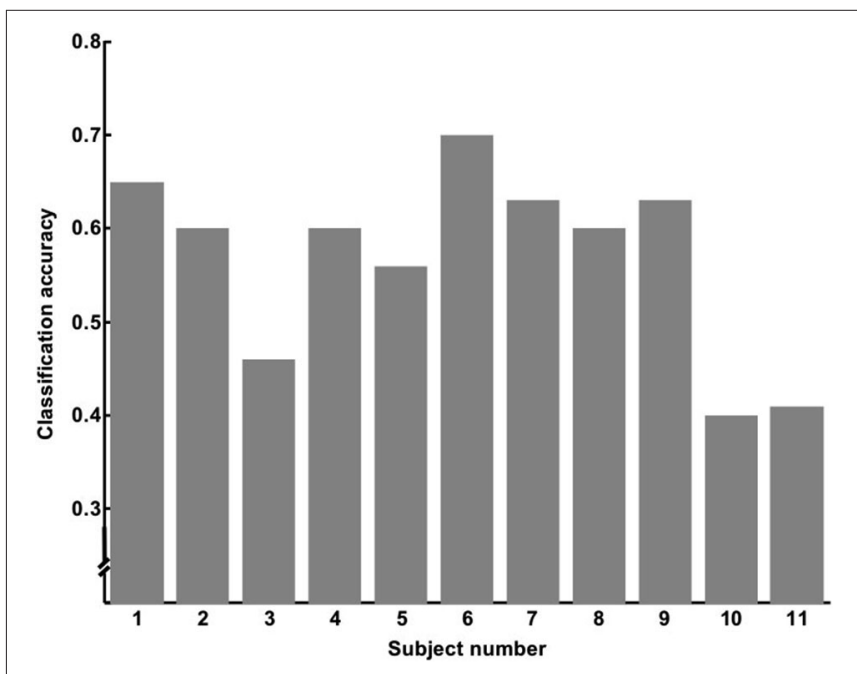

FIGURE 6 | Accuracy per subject obtained for classification of SART Error vs. SART No Error trials.

et al., 2009; Stevenson et al., 2011). Nevertheless, previous investigations including both SART and experience sampling experiments performed online support that SART errors are linked to MW (Christoff et al., 2009). In addition, one may argue that our results on SART errors trials could correspond to the absence of motor response inhibition. Despite the high rate of retrospective assessment of MW, the occurrence of SART Errors even without meta-awareness during the experiment suggests that MW alone cannot account for the totality of the SART Errors. Nevertheless, further investigation is needed concerning the presence or absence of left DLPFC activations, as the voxels in this area had to be removed, and to confirm that the activations measured relate only to MW. 
Classification accuracy showed that single-trial classification returned relatively poor results, although significantly better than chance, indicating that real time detection of such events using only fNIRS signal would be difficult to achieve. These results could in part be due to the high intersubject variability observed with fNIRS signals (Jasdzewski et al., 2003; Sato et al., 2005). Moreover, the temporal closeness between target trials, due to their rate among all the trials (11\%) may have potentially jeopardized single-trial classification. Although further investigation using a modified protocol is needed to eliminate the potential confound, the low classification accuracy we obtained does not make fNIRS a good candidate to detect MW in real time, when used alone. Nevertheless, the significance of the classification compared

\section{References}

Allan Cheyne, J., Solman, G. J., Carriere, J. S., and Smilek, D. (2009). Anatomy of an error: a bidirectional state model of task engagement/disengagement and attention-related errors. Cognition 111, 98-113. doi: 10.1016/j.cognition.2008. 12.009

Braboszcz, C., Hahusseau, S., and Delorme, A. (2010). "Meditation and neuroscience: from basic research to clinical practice," in Integrative Clinical Psychology, Psychiatry and Behavioral Medicine: Perspectives, Practices and Research, ed R. Carlstedt (New York: Springer Publishing), 1910-1929.

Casner, S. M., and Schooler, J. W. (2014). Thoughts in flight automation use and pilots task-related and task- unrelated thought. Hum. Factors 56, 433-442. doi: 10.1177/0018720813501550

Christoff, K., Gordon, A. M., Smallwood, J., Smith, R., and Schooler, J. W. (2009). Experience sampling during FMRI reveals default network and executive system contributions to mind wandering. Proc. Natl. Acad. Sci. U S A 106, 8719-8724. doi: 10.1073/pnas.0900234106

Crawford, J. R., and Garthwaite, P. H. (2007). Comparison of a single case to a control or normative sample in neuropsychology: development of a Bayesian approach. Cogn. Neuropsychol. 24, 343-372. doi: 10.1080/026432907012 90146

Delorme, A., and Makeig, S. (2004). Eeglab: an open source toolbox for analysis of single-trial eeg dynamics including independent component analysis. J. Neurosci. Methods 134, 9-21. doi: 10.1016/j.jneumeth.2003.10.009

Galéra, C., Orriols, L., M’Bailara, K., Laborey, M., Contrand, B., Ribéreau-Gayon, R., et al. (2012). Christmas 2012: research: mind wandering and driving: responsibility case-control study. BMJ 345:e8105. doi: 10.1136/bmj.e8105

Grandchamp, R., Braboszcz, C., and Delorme, A. (2014). Oculometric variations during mind wandering. Front. Psychol. 5:31. doi: 10.3389/fpsyg.2014. 00031

Gruberger, M., Ben-Simon, E., Levkovitz, Y., Zangen, A., and Hendler, T. (2011). Towards a neuroscience of mind-wandering. Front. Hum. Neurosci. 5:56. doi: 10.3389/fnhum.2011.00056

Harrivel, A. R., Weissman, D. H., Noll, D. C., and Peltier, S. J. (2013). Monitoring attentional state with fNIRS. Front. Hum. Neurosci. 7:861. doi: 10.3389/fnhum. 2013.00861

He, J., Becic, E., Lee, Y.-C., and McCarley, J. S. (2011). Mind wandering behind the wheel performance and oculomotor correlates. Hum. Factors 53, 13-21. doi: 10. 1177/0018720810391530

Helton, W. S., Kern, R. P., and Walker, D. R. (2009). Conscious thought and the sustained attention to response task. Conscious. Cogn. 18, 600-607. doi: 10. 1016/j.concog.2009.06.002

Helton, W. S., Ossowski, U., and Malinen, S. (2013). Post-disaster depression and vigilance: a functional near-infrared spectroscopy study. Exp. Brain Res. 226, 357-362. doi: 10.1007/s00221-013-3441-4

Jasdzewski, G., Strangman, G., Wagner, J., Kwong, K., Poldrack, R., and Boas, D. (2003). Differences in the hemodynamic response to event-related motor and visual paradigms as measured by near-infrared spectroscopy. Neuroimage 20, 479-488. doi: 10.1016/s1053-8119(03)00311-2 to chance suggests that the fNIRS signal could complement other methodologies such as pupil diameter (Grandchamp et al., 2014) or electro-encephalography (Braboszcz et al., 2010) to improve classification performance, and predict MW before subjects become aware of them (O'Connell et al., 2009).

\section{Acknowledgments}

The study was funded by the Neurocockpit project of French Region Midi Pyrenees, and Pole de Recherche et d'Enseignement Superieur. The authors would like to thank Marc Mari-Mari and Alvaro Miedes for their help during data collection, and Tracy Brandmeyer for her help with editing.

Kennedy, A. D., and Gehan, E. A. (1971). Computerized simple regression methods for survival time studies. Comput. Programs Biomed. 1, 235-244. doi: $10.1016 / 0010-468 \times(71) 90014-6$

Killingsworth, M. A., and Gilbert, D. T. (2010). A wandering mind is an unhappy mind. Science 330:932. doi: 10.1126/science.1192439

Mackworth, N. H. (1948). The breakdown of vigilance durning prolonged visual search. Q. J. Exp. Psychol. 1, 6-21. doi: 10.1080/17470214808416738

Manly, T., Robertson, I. H., Galloway, M., and Hawkins, K. (1999). The absent mind: further investigations of sustained attention to response. Neuropsychologia 37, 661-670. doi: 10.1016/s0028-3932(98)00127-4

Maris, E., and Oostenveld, R. (2007). Nonparametric statistical testing of EEG-and MEG-data. J. Neurosci. Methods 164, 177-190. doi: 10.1016/j.jneumeth.2007. 03.024

Mason, M. F., Norton, M. I., Van Horn, J. D., Wegner, D. M., Grafton, S. T., and Macrae, C. N. (2007). Wandering minds: the default network and stimulusindependent thought. Science 315, 393-395. doi: 10.1126/science.1131295

Mooneyham, B. W., and Schooler, J. W. (2013). The costs and benefits of mind-wandering: a review. Can. J. Exp. Psychol. 67, 11-18. doi: 10.1037/a00 31569

O’Connell, R. G., Dockree, P. M., Robertson, I. H., Bellgrove, M. A., Foxe, J. J., and Kelly, S. P. (2009). Uncovering the neural signature of lapsing attention: electrophysiological signals predict errors up to $20 \mathrm{~s}$ before they occur. J. Neurosci. 29, 8604-8611. doi: 10.1523/JNEUROSCI.596708.2009

Peterson, B., Potenza, M., Wang, Z., Zhu, H., Martin, A., Marsh, R., et al. (2009). An FMRI study of the effects of psychostimulants on default-mode processing during stroop task performance in youths with ADHD. Am. J. Psychiatry 166, 1286-1294. doi: 10.1176/appi.ajp.2009.08050724

Sämann, P. G., Wehrle, R., Hoehn, D., Spoormaker, V. I., Peters, H., Tully, C., et al. (2011). Development of the brain's default mode network from wakefulness to slow wave sleep. Cereb. Cortex 21, 2082-2093. doi: 10.1093/cercor/bhq295

Sasai, S., Homae, F., Watanabe, H., Sasaki, A. T., Tanabe, H. C., Sadato, N., et al. (2012). A NIRS-FMRI study of resting state network. Neuroimage 63, 179-193. doi: 10.1016/j.neuroimage.2012.06.011

Sato, H., Fuchino, Y., Kiguchi, M., Katura, T., Maki, A., Yoro, T., et al. (2005). Intersubject variability of near-infrared spectroscopy signals during sensorimotor cortex activation. J. Biomed. Opt. 10:044001. doi: 10.1117/1. 1960907

Shaw, T. H., Funke, M. E., Dillard, M., Funke, G. J., Warm, J. S., and Parasuraman, R. (2013). Event-related cerebral hemodynamics reveal target-specific resource allocation for both go and no-go response-based vigilance tasks. Brain Cogn. 82, 265-273. doi: 10.1016/j.bandc.2013.05.003

Smallwood, J., and Andrews-Hanna, J. (2013). Not all minds that wander are lost: the importance of a balanced perspective on the mind-wandering state towards a balanced perspective of the mind-wandering state. Front. Psychol. 4:441. doi: 10.3389/fpsyg.2013.00441

Smallwood, J., Beach, E., Schooler, J. W., and Handy, T. C. (2008). Going AWOL in the brain: mind wandering reduces cortical analysis of external events. J. Cogn. Neurosci. 20, 458-469. doi: 10.1162/jocn.2008.20037 
Smallwood, J., and Schooler, J. W. (2006). The restless mind. Psychol. Bull. 132, 946-958. doi: 10.1037/0033-2909.132.6.946

Stawarczyk, D., Majerus, S., Maquet, P., and D'Argembeau, A. (2011). Neural correlates of ongoing conscious experience: both task-unrelatedness and stimulus-independence are related to default network activity. PLoS One 6:e16997. doi: 10.1371/journal.pone.0016997

Stevenson, H., Russell, P. N., and Helton, W. S. (2011). Search asymmetry, sustained attention and response inhibition. Brain Cogn. 77, 215-222. doi: 10. 1016/j.bandc.2011.08.007

Strait, M., and Scheutz, M. (2014). What we can and cannot do with near infrared spectroscopy. Front. Neurosci. 8:117. doi: 10.3389/fnins.2014.00117

Szpunar, K. K., Moulton, S. T., and Schacter, D. L. (2013). Mind wandering and education: from the classroom to online learning. Front. Psychol. 4:495. doi: 10. 3389/fpsyg.2013.00495
Warm, J. S., Matthews, G., and Parasuraman, R. (2009). Cerebral hemodynamics and vigilance performance. Mil. Psychol. 21, S75-S100. doi: 10.1080/ 08995600802554706

Conflict of Interest Statement: The authors declare that the research was conducted in the absence of any commercial or financial relationships that could be construed as a potential conflict of interest.

Copyright (C) 2015 Durantin, Dehais and Delorme. This is an open-access article distributed under the terms of the Creative Commons Attribution License (CC BY). The use, distribution and reproduction in other forums is permitted, provided the original author(s) or licensor are credited and that the original publication in this journal is cited, in accordance with accepted academic practice. No use, distribution or reproduction is permitted which does not comply with these terms. 